\title{
A MEASURABLE MATHEMATICAL MODEL FOR PROCESSES
}

\author{
MIN MA ${ }^{1,2}$ and FRED VAN OYSTAEYEN ${ }^{1}$ \\ 1Department of Mathematics and Computer Science \\ University of Antwerp \\ Belgium \\ e-mail: mamin_psych@163.com \\ 2Department of Psychology \\ Central University of Finance and Economics \\ P. R. China
}

\begin{abstract}
We present a generic mathematical model for describing processes evolving in time. The method we propose is a "deformation" of a process described on a set of process "indicators" obtained by a description of each indicator by a set of "aspects" allowing the definition and study of the microstructure of the process. We define a process as a set of indicators with a relation. There is the set of aspects with a (deformed) relation and a valuation function, which can be used to reconstruct the indicator relation from the aspect relation. The valuation function in the structure provides the measurable property. It can be based on questionnaires in more social processes or an accurate scientific measurement in concrete physical processes or technological processes.
\end{abstract}

\section{Introduction}

We present a rather generic mathematical model for describing processes evolving in time. The method we propose is a "deformation" of a process described on a set of process "indicators" obtained by a 2010 Mathematics Subject Classification: 03.

Keywords and phrases: processes, deformation, indicators, aspects, valuation function.

Received June 22, 2015

(ㄷ) 2015 Scientific Advances Publishers 
description of each indicator by a set of "aspects" allowing the definition and study of the microstructure of the process. We look at the global process as being a series of momentary processes evolving in time, here a purely mathematical glueing result (Proposition 2.5.3) allows to view the evolution of the process from the "endpoint" situation, i.e., when the process has stopped. Then we can define a process as first a set of indicators with a relation, in fact any relation may be considered but for practical applications a partial order relation, e.g., a causal relation, in most interesting. Secondly, there is the set of aspects with a (deformed) relation and a valuation function, say to real numbers, that can be used to reconstruct the indicator relation from the aspect relation. This structure is called the deformed process or micro-process describing the indicator process. The aspect relation need not be a partial order even if it defines a partial order or the indicator set; an important example of this is the learning process [1], where non-causal relation representing input from fantasy are important in the micro-structure of the learning process. Similar applications appear in the creativity process [1].

The valuation function in the structure provides the measurable property; it can be based on questionnaires in more social processes or an accurate scientific measurement in concrete physical processes or technological processes. In fact, one may start from a theoretical function (educated guess, ideal situation, a selected measurement) and compare consequent measuring to the fixed gauge model in order to study the evaluation of the process in different circumstances or time frames. The measurable study of micro-structure of a process seems to allow many different applications, from the pollution of a river, biodiversity in some biotopes, to safety of production processes in industry,.... We only mention some possibilities here, several outside our field of expertise (but some applications are in our studies on learning process and creativity process [1]), hoping that the new structure of "deformed indicator process" or micro-process may find many applications in different fields. 


\section{Formalization of Processes and Micro-structure}

\subsection{Generalities}

We view a process in time as a creation (in time) of relations on a global set of indicators, $I$ say, describing the process, $P$ say, globally, i.e., as looking back from its finished state. The selection of a set of indicators has some freedom but of course it has to be done with some care in practice, the description of a process in reality has to provide a good approximation of the real phenomena. There may be more than one relation on the set of indicators but we will present the theory for one relation, then in case more relations have to be considered you have to repeat the constructions for each relation separately but that is straight forward.

Deeper understanding of the process depends on a deeper analysis of the indicators by considering, components, technical properties, behaviour for each indicator. We therefore describe each indicator $A$ from $I$ by a set of so-called aspects $a_{1}, \ldots, a_{n}$ of the indicator $A$. For example, aspects of a cube could be its edges, faces, symmetries, inscribed sphere, ...; aspects of the level of cadmium in a river could be the level of cadmium in the land near the river or the level of cadmium in the river in certain period of a year. Let us call $\mathcal{A}$ the aspect set for the indicator set $I$ of a process $P$. We then have a surjective map, $\pi: \mathcal{A} \rightarrow I$, where for an $A \in I$ the set of aspects of $A$ is exactly $\pi^{-1}(A)$ in $\mathcal{A}$. We shall consider $A$ as the set of its aspects and write $a \in A$ meaning $a \in \pi^{-1}(A)$, this creates no ambiguity. The logical structure of the process is encoded in a relation $q$ defined on $\mathcal{A}$. Result that a relation is a subset of the product set $\mathcal{A} \times \mathcal{A}$ and we write $a q b$ if $(a, b) \in q$ for some $a, b \in \mathcal{A}$. It is useful to use the graphical notation $a \rightarrow b$ to denote $(a, b) \in q$. In case $q$ is a causal relation, then $a \rightarrow b$ would just mean $a$ implies $b$. The process viewed on indicators only will also induce a relation $R$ on $I$ and $R$ will be related to $q$ in some numerical way to be explained later. 
The aspect set with the aspect relation defines a kind of microstructure, i.e., the aspect description allows you to understand the origin of the relation $R$ via the finer aspect description. In case $R$ is a partial order, e.g., a causal relation, $q$ does not need to be, hence the microstructure in this case reveals non-casual aspects (cf. learning or the creativity process [1]). The microstructure of the process may be seen as a deformation (similar as in Algebraic Geometry or Noncommutative Geometry [2,3]) and to do this in a "measurable" way we evaluate the aspect process by giving a weight (for example, a measure for the importance of the aspect in the description of the process) to each aspect. In fact, this will lead to a weight on relations between aspects in a traceable way.

So we assume given an aspect weight: $\omega: \mathcal{A} \rightarrow R_{+}$, where $R_{+}$ stands for the non-negative real number. We also allow aspects with weight zero but they play no role in the static theory, however in the dynamic theory aspects may arise or vanish in time and so it is good to introduce them in the global process but with weight zero in the momentary processes at times where they not present. We will give a numerical rule to construct an indicator relation $R$ from an aspect relation $q$ with respect to an aspect weight function.

\subsection{Aspect relation and indicator relation}

Given $\pi$ then $\left\{\pi^{-1}(A), A \in I\right\}$ is a partition of $\mathcal{A}$ if $A \neq B \in I$, then $\pi^{-1}(A)$ and $\pi^{-1}(B)$ are disjoint and together the $\pi^{-1}(A)$ for $A$ varying over $I$ cover $\mathcal{A}$; conversely, it is clear that every partition of $\mathcal{A}$ defines a surjection to a specific indicator set.

Definition 2.2.1. We say that $q$ is an aspect relation if

(1) $q$ is reflexive : aqa, for all $a \in \mathcal{A}$.

(2) $q$ separates indicators : if $a q b$ with $a, b \in A$, then $a=b$, or if $a \neq b$ in $\mathcal{A}$, then $a q b$ excludes that $a$ and $b$ belong to the same indicator. 
In practical examples, several functions on $\mathcal{A}$ appear naturally, e.g., weights, probabilities, gradings, importance, intensity. We define an aspect weight as a map $w: \mathcal{A} \rightarrow \mathbb{R}_{+}$, where $\mathbb{R}_{+}$stands for the nonnegative real numbers. An aspect relation weight is an aspect weight such that $a q b$ entails $w(a) \geq w(b)$, we shall abbreviate this to $a r$-weight. If for an $a r$-weight $w$ we have $w(a)>w(b)$, then there cannot exist a path starting at $b$ and ending at $a$, i.e., $b \rightarrow \ldots \rightarrow \ldots a$. Some more notation : for $a \in \mathcal{A}$, we let the target set be $T(a)=\{b \in \mathcal{A}, a q b\}$, the origin set is then $O(a)=\{c \in \mathcal{A}$, eqa $\}$. We say that $(\mathcal{A}, q, I)$ is finitary if for every $a \in \mathcal{A}$, the sets $T(a)$ and $O(a)$ are finite sets and $I$ defines a partition consisting of finite sets. In this work, we restrict attention to the finitary case. This is important with respect to some weight calculations later.

Definition 2.2.2. Given $q$ and an aspect weight $w: \mathcal{A} \rightarrow \mathbb{R}_{+}$we define a relation $\hat{q}$ on $Q(\mathcal{A})$, the set of subsets of $\mathcal{A}$, by putting $X \widehat{q} Y$ for subsets $X$ and $Y$ of $\mathcal{A}$, if either $Y=X$ or else $Y \neq X$ and the following holds : $\delta_{q}(X \times Y)>0$, where $\delta_{q}$ is the diagonal weight defined as follows. First, we define the graph-weight of a subset $S$ of $\mathcal{A} \times \mathcal{A}$ by looking at $S \cap G(q)$, where $G(q)=\{(a, b) \in \mathcal{A} \times \mathcal{A}, a q b\}$ is the graph of $q$ and putting:

$$
w_{q}(S)=\left(\sum_{S \cap G(q)} w(a) w(b)\right)\left(\sum_{S} w(a) w(b)\right)^{-1}
$$

where for any $X \subset \mathcal{A} \times \mathcal{A}$ we use notation $\sum_{X}$ to indicate a sum over all $(a, b) \in X$.

Now, we associate to $S \subset \mathcal{A} \times \mathcal{A}$ the opposite $S^{o}, S^{o}=\{(x, y),(y, x) \in S\}$ and the diagonal weight $\delta_{q}$ is given by : $\delta_{q}(S)=w_{q}(S)-w_{q}\left(S^{o}\right) \in \mathbb{R}$. We say that $S$ is q-oriented if $\delta_{q}(S) \in \mathbb{R}_{+}$and $S$ is said to be well-oriented if it is $q$-oriented and $\delta_{q}(S) \neq 0$. The definition above then translates to : $X \widehat{q} Y$, for $X \neq Y$, when $X \times Y$ is well-oriented in $\mathcal{A} \times \mathcal{A}$. 
Observe also that we may extend $w: \mathcal{A} \rightarrow \mathbb{R}_{+}$to a map $: \widehat{w}: Q(\mathcal{A}) \rightarrow \mathbb{R}_{+}$ by putting for $X \subset \mathcal{A}, \widehat{w}(X)=\sum_{x \in X} w(x)$. Note that $w$ can be recovered from $\widehat{w}$ because $\widehat{w}(\{a\})=w(a)$. For an indicator $A \in I$, let us write $w(A)$ for $\sum_{a \in A} w(a)$. In particular, if $S=\pi^{-1}(A) \times \pi^{-1}(B)$ with $A \neq B$ in $I$, then we write $S=S(A, B)$ and

$$
\begin{gathered}
w_{q}\left(\pi^{-1}(A) \times \pi^{-1}(B)\right)=w_{q}(A, B), \\
w_{q}(A, B)=\left(\sum_{S \cap G(q)} w(a) w(b)\right) w(A)^{-1}(B)^{-1},
\end{gathered}
$$

because $\pi^{-1}(A)$ and $\pi^{-1}(B)$ are disjoint sets in $(\mathcal{A})$.

Definition 2.2.3. The indicator relation $R$ induced by $q$ and the aspect weight $w$ is defined by putting $A R B$ if and only if $\pi^{-1}(A) \widehat{q} \pi^{-1}(B)$.

Observe that $X \widehat{q} Y$ for $X \neq Y$ means exactly

$$
\sum_{(X \times Y) \cap G(q)} w(x) w(y)>\sum_{(Y \times X) \cap G(q)} w\left(y^{\prime}\right) w\left(x^{\prime}\right) .
$$

Proposition 2.2.4. The relation $R$ is reflexive and antisymmetric on $I$.

Proof. Reflexivity of $R$ follows by definition. Suppose that for some $A \neq B$ in $I: A R B$ and $B R A$, then $\delta_{q}\left(\pi^{-1}(A) \times \pi^{-1}(B)\right)=0$ but that is excluded since $\pi^{-1}(A) \hat{q} \pi^{-1}(B)$ with $A \neq B$ makes $\pi^{-1}(A) \times \pi^{-1}(B)$ well oriented.

Definition 2.2.5. In case $R$, as defined above, is also transitive, then $R$ defines a partial order on $I$ and $q$ is said to be indicator ordering via $w$. We may thing of $q$ as a deformation of the partial order relation $R$ and refer to it as a $w$-deformation of $R$. Transitivity of $R$ does not entail transitivity of $q$ or $\hat{q}$, but suitable path-wise properties may be discussed. 
In order to define process-units in $I$, we shall call them 1-clusters, we need the existence of maximal chains of relations established in the following theorem.

\subsection{The cluster process}

In order to define clusters (1-clusters) in $P$, we need the existence of maximal chains of relations. A path in $I$ between $A_{1}$ and $A_{n}$, say $A_{1} \rightarrow$ $A_{2} \rightarrow \ldots \rightarrow A_{n}$ is said to be irreducible if it is not part of a longer path connecting $A_{1}$ and $A_{n}$. Hence the relation $A \rightarrow B$ is irreducible if there is no $C \neq A, B$ such that: $A \rightarrow C \rightarrow B$.

Theorem 2.3.1. Let $(\mathcal{A}, q, I)$ be a finitary process with indicator relation $R$ (also written $\rightarrow$ ) being also transitive, thus a partial order. For any $A, B \in I$ such that $A \rightarrow B$, the path $A \rightarrow B$ can be refined to a finite irreducible path. (This is of course easy to see for a finite process.)

Proof. Let us write $i(A, B)$ for the subset of $I$ consisting of $C \in I$ such that we have $A \neq C$ and

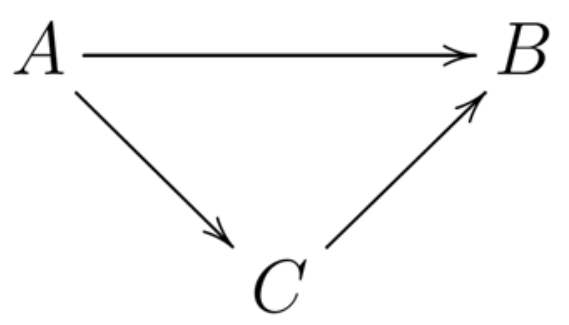

In case $i(A, B)$ is an infinite set, then there are infinitely many different $C_{\alpha}$ with $A \rightarrow C_{\alpha}$. For every $\alpha$, there is an $a_{\alpha} \in A$ such that for some $c_{\alpha} \in C_{\alpha}$ we have $a_{\alpha} \rightarrow c_{\alpha}$, where $x_{\alpha} \in C_{\alpha}$ are all different. Finiteness of $\pi^{-1}(A)$ yields that for some fixed $a_{0} \in A$ there are infinitely many $a_{0} \rightarrow c_{\alpha^{\prime}}$ with all $c_{\alpha^{\prime}}$ being different. However $T\left(a_{0}\right)$ is finite and each $c_{\alpha^{\prime}} \in T\left(a_{0}\right)$, thus we reach a contradiction or consequently if $A R B$, then $i(A, B)$ is finite, hence there is an $A_{1} \in I$, in fact $A_{1}$ is one of the intermediates between $A$ and $B, A \rightarrow A_{1} \rightarrow B$, such that $A \rightarrow A_{1}$ 
cannot be refined. Now for $A_{1} \rightarrow B$, we repeat the argument and find $A_{2} \in I$ such that $A_{1} \rightarrow A_{2}$ is irreducible and $A_{2} \rightarrow B$ (uses transitivity) etc. until we reach $A \rightarrow A_{1} \rightarrow A_{2} \rightarrow \ldots \rightarrow A_{\alpha} \rightarrow B$, where we may suppose all of these different and $A_{i} \mapsto A_{i+1}$ irreducible.

For any $\alpha$, there are $a_{\alpha} \in A_{\alpha}$ and $b_{\alpha} \in B$ such that $a_{\alpha} \rightarrow b_{\alpha}$. The finiteness of $\pi^{-1}(B)$ yields that for some fixed $b_{0} \in B$ there must be infinitely many $a_{\alpha} \rightarrow b_{0}$ if there were infinitely many possible $A_{\alpha}$ in the construction. By assumption $O\left(b_{0}\right)$ is finite, hence there can only be finitely many $A_{\alpha}$ in the construction:

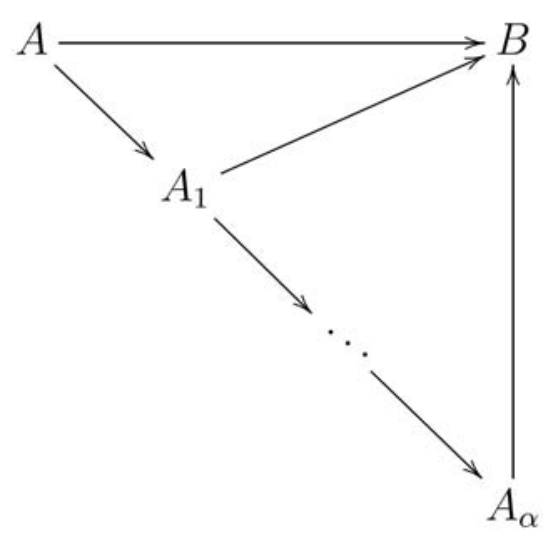

At the moment we reach an irreducible $A_{\alpha} \rightarrow B$, we also obtain a chain $A \rightarrow \ldots \rightarrow A_{\alpha} \rightarrow B$ where every arrow is irreducible, hence we reach an irreducible path from $A$ to $B$ of finite length.

Observe that there may be several irreducible paths from $A$ to $B$ in $I$.

Corollary 2.3.2. For every $A \in I$, we may define the cluster of $A$ by putting $T^{1}(A)=\{B \in I, A \rightarrow B$ is irreducible $\}$. An $A \in I$ is said to be primary if $0(A)=A$, i.e., there are no (nontrivial) $X \rightarrow A$ for $X \in I,(X \neq A)$. Any irreducible path from $A$ to $B$ in $I$ may be seen as a sequence $A_{0}=A \rightarrow A_{1} \rightarrow \ldots \rightarrow A_{n}=B$, where $A_{j} \in T^{1}\left(A_{j-1}\right)$ for $j=1, \ldots, n$. The $R$-distance $d(A, B)$ is the number of $n$ for which there is 
an irreducible path $A_{0}=A \rightarrow \ldots \rightarrow A_{n}=B$ above such that it is minimal between the possible lengths of irreducible paths. Another corollary of the theorem is that $\left|T^{1}(A)\right|$ is finite and for every $A \rightarrow B$ there exists at least one $A_{i} \in T^{1}(A)$ such that $A_{i} \rightarrow B$.

In most practical situations, one will have to work with finite processes. However, often the duration of the process may be unknown, arbitrary long, so then one has to treat it as a finitary process and the foregoing and following are established therefore in that theoretical generality.

Definition 2.3.3. Consider the clusters $\left\{T^{1}(A), A \in I\right\}$ as the objects of a new process $\mathcal{L}(P)$ termed the learning process of $P$ (it encodes the objects of $P$ plus the knowledge of primary connotations between objects), where we define $T^{1}(A) R T^{1}(B)$ if and only if $A R B$. Then $\mathcal{L}(P)$ is again a finitary process. Observe that for $B$ and $C$ in $T^{1}(A), A \neq B, A \neq C$, we have $B R C$ if and only if $B=C$ because of the diagram

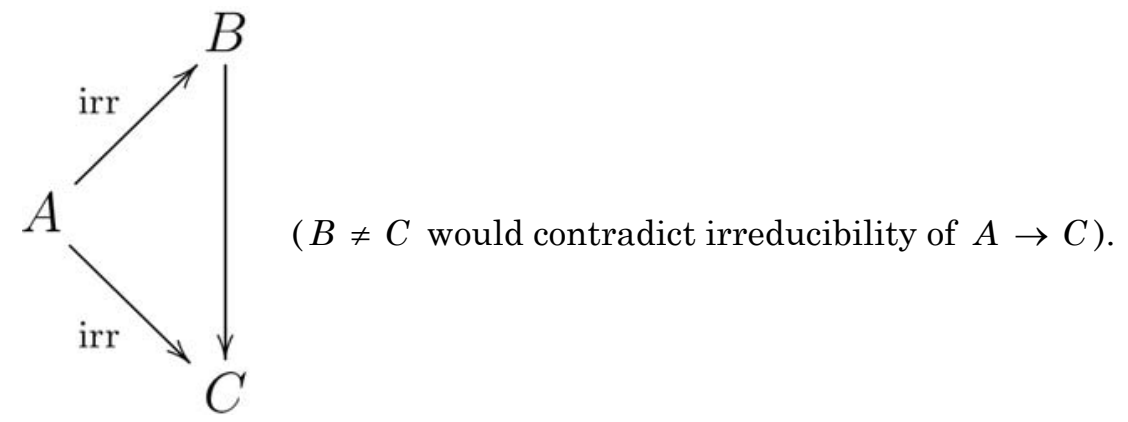

A hypothesis weaker than transitivity for $R$, i.e., $R$ would then be a partial ordering of $I$, is the reducedness of $R$, obtained by demanding that there do not exist finite loops for $R$, i.e., no nontrivial $A \rightarrow A_{1} \rightarrow \ldots$ $\rightarrow A_{d} \rightarrow A$.

Definition 2.3.4. We define the deep learning process of $P$, denoted $\mathcal{D}(P)$, taking the 1-clusters of $P$ as the indicator set (in fact this corresponds bijectively to the original indicator set because every $A \in I$ 
is unambiguously determined by its cluster $T^{1}(A)$ as $A$ is the unique primary element in $T^{1}(A)$. The definition of the relation $R^{\mathcal{D}}$ is obtained stepwise. We say that $T^{1}(A) R_{-1}^{\mathcal{D}} T^{1}(B)$ if $A \rightarrow B$ is irreducible and for every $B_{1} \in T^{1}(B)$ there exists $A_{i} \in T_{A}^{1}$ such that $A_{i} \rightarrow B_{i}$. Then $T^{1}(C) R_{-2}^{\mathcal{D}} T^{1}(B)$ if there exists $T^{1}(A)$ such that $T^{1}(C) R_{-1}^{\mathcal{D}} T^{1}(A)$ and also $T^{1}(A) R_{-1}^{\mathcal{D}} T^{1}(B)$. Clearly, $R^{\mathcal{D}}$ is defined by the existence of a chain $T^{1}\left(B_{n}\right)=T^{1}(A) \rightarrow T^{1}\left(B_{n-1}\right) \rightarrow \cdots \rightarrow T^{1}(B) \quad$ where each $T^{1}\left(B_{i}\right) \rightarrow T^{1}\left(B_{i-1}\right)$ represents a relation $R_{-1}^{\mathcal{D}}$, which is by definition associated to an $R$-chain $A=B_{n} \rightarrow B_{n-1} \rightarrow \cdots \rightarrow B$, where each arrow is irreducible for $R$. We say that $T(A) R^{\mathcal{D}} T(B)$ if there exists a finite $n$ such that $T(A) R_{-n}^{\mathcal{D}} T(B)$. For now, we do not go into the definition of the aspect deformation. If for some process no aspects are being considered then we adapt the definition of finitary by only demanding that target sets and original sets are finite.

Lemma 2.3.5. If $P$ is finitary such that $R$ is reduced then $R^{\mathcal{D}}$ is finitary and reduced.

Proof. Clearly $R^{\mathcal{D}}$ is reflexive; we also get antisymmetry because of $T^{1}(A) R^{\mathcal{D}} T^{1}(B)$ as well as $T^{1}(B) R^{\mathcal{D}} T^{1}(A)$, then we have $R$-chains $A \rightarrow B_{n-1} \rightarrow \ldots \rightarrow B \rightarrow A_{m-1} \rightarrow \ldots \rightarrow A$ which will be a nontrivial loop for $R$.

Suppose that $T^{1}\left(A_{1}\right) \rightarrow \ldots \rightarrow T_{1}\left(A_{n}\right)$, with $T^{1}\left(A_{1}\right)=T^{1}\left(A_{n}\right)$, is a loop for $R^{\mathcal{D}}$, then it is obvious that we arrive at a loop for $R: A_{1} \rightarrow \ldots \rightarrow A_{n}=A_{1}$.

The choice of terminology suggests that the cluster process has applications in describing formally some didactical aspects of learning processes, we treat this elsewhere. 


\subsection{Variations on indicator weights}

Let us return to aspect weights. We define

$$
t(a)=\sum_{x \in T(a)} w(a) w(x), \quad o(b)=\sum_{y \in O(b)} w(y) w(b) .
$$

The relative weight of $a q b$, denoted by $w(a, b)$ is: $w(a, b)=w(a) w(b)$ $t(a)^{-1}$. Obviously, we have that $\sum_{x \in T(a)} w(a, x)=1$ and all $w(a, b) \in$ $[0,1]$.

Similarly, the historic weight of $a q b$, denoted by $h(a, b)$ is: $h(a, b)=w(a) w(b) o(b)^{-1}$.

The case where $w$ is referred to as the unweighted case; then $w(a, b)$ $=v(a)^{-1}$, where $v(a)=|T(a)|, h(a, b)=s(b)^{-1}$, where $s(b)=|O(b)|$. In the un-weighted case earlier defined weights reduce to: $w_{q}(A, B)=$ $|q(A, B)| n_{A}^{-1} n_{B}^{-1}$, where we put $n_{A}=\left|\pi^{-1}(A)\right|$ for every $A \in I$ and $q(A, B)$ is the number of arrows from aspects of $A$ to aspects of $B$ or conversely.

The relative weight of $a q b$ with $a \in A, b \in B$ may be modified in several ways so as to account for certain properties of the indicator partition. First observe that we put $w(x, y)=0$ whenever $(x, y) \notin q$, so we do need to look at the weight on a relation as a map to $\mathbb{R}_{+}$as we defined it (including the zero).

\subsubsection{Exercise and project}

In some concrete applications, the validity of a relation $a q b$ may be depending on a probability, so we are given $p: G(q) \mapsto[0,1]$. The relative weight $w(a, b)$ can then be replaced by the probable weight $p(a, b) w(a, b)$ and $w_{q}(A \times B)$ is replaced by $p w_{q}(A \times B)$ given by 


$$
\left(\sum_{(A \times B) \cap G(q)} p(a, b) w(a) w(b)\right) w(A)^{-1} w(B)^{-1},
$$

where by the probable diagonal weight appears as $p \delta_{q}(S)=p w_{q}(S)-$ $p w_{q}(S)^{0}$. The probable indictor relation $R^{p}$ follows from $p \delta_{p}$ just as before of $R$. Small variations in the probability function may even lead up to an unchanged relation on indicators, this allows certain manipulations on the process ingredients and can best be formulated in the context of interactive processes focused on later in this text. Probabilistic development of the whole process description and evaluation is left here as a project. The process with its intrinsic structure may itself be seen as a statistical variable, moreover part of a dynamical evaluation in time, requiring a new statistical approach (fixing some specific structure properties, e.g., the indicator relation).

\subsubsection{Indicator weight}

For $a q b$ with $a \in A, b \in B$ and $A, B \in I$, we define the indicator weight as follows: $i w(a, b)=w(a) w(b) t_{B}(a, b)^{-1}$, where $t_{B}(a, b)=$ $\sum_{B \cap T(a)} w(a) w(x)$ and $B \cap T(a)$ is a finite set with $|B \cap T(a)|=v_{B}(a)$.

In the unweighted situation, we would thus have $i w(a, b)=v_{B}(a)^{-1}$. The historic indicator weight is defined similarly, $i h(a, b)=w(a) w(b) o_{A}(a, b)^{-1}$, where $o_{A}(a, b)=\sum_{A \cap O(b)} w(x) w(b)$ and $A \cap O(b)$ is a finite set with $|A \cap O(b)|=s_{A}(b)$ (source $b$ in $A$ ). For these indicator weights, we have

$$
\sum_{B \cap T(a)} i w(a, x)=1=\sum_{A \cap O(b)} i h(x, b) .
$$

In case we have an indicator ordering $\mathcal{A}$, $q$, i.e., $R$ is a partial order on $I$, then for each $A \in I$ only finite many indicators $X \in I$ are order comparable to $A$. In many practical situations, $I$ is finite anyway and foregoing effect of the finitely assumption is trivial. For $A, B$ in $I$, we express the dependency of $B$ on $A$, or the contribution of $A$ to $B$, as follows: 


$$
\begin{aligned}
d(A, B) & =\left(\sum_{A} t_{A}(x, b)\right)\left(\sum_{A} t(x)\right)^{-1} \\
& =\left(\sum_{(A \times B) \cap G(q)} w(a) w(b)\right)\left(\sum_{(a, x) \in q} w(a) w(x)\right)^{-1} ; \\
c(A, B) & =\left(\sum_{B} O_{B}(a, x)\right)\left(\sum_{B} o(b)\right)^{-1} \\
& =\left(\sum_{(A \times B) \cap G(q)} w(a) w(b)\right)\left(\sum_{(x, b) \in q} w(x) w(b)\right)^{-1} .
\end{aligned}
$$

Replacing $w(a, b)$ by $i w(a, b)$, one may construct $i \widehat{q}$ as well as $i R$ in a way completely similar to the construction of $\widehat{q}$ and $R$. The indicator weight depends on the relative importance of $B$ compared to other indicators expressed in terms of "size" of intersection of $\pi^{-1}(B)$ and $T(a)$ for $a \in A$; however, a weight associated to $A R B$ should preferably only depend on $A$ and $B$ and not on their indicators. Therefore, it is natural to introduce the dominant weight of $A R B$ by putting: $d w(A, B)=\delta_{q}\left(\pi^{-1}(A)\right.$, $\left.\pi^{-1}(B)\right)$ when $A \neq B$ and putting $d w(A, A)=1$. On the other hand, if $(I, R, W)$ derives from $(\mathcal{A}, q, w)$ as explained above, now writing $W$ for the indicator weight, then we may define all versions of weights and related notions, introduced earlier, directly to $(I, R, W)$. In doing so, we arrive at a relative weight for $A R B$ expressed as: $W(A) W(B) t(A)^{-1}$, with $t(A)=\sum_{T(A)} W(A) W(X)$. The latter is obviously different from the dominant weight introduced before because that is calculated taking into account the aspect relation, i.e., the way $R$ has been defined from $q$. Therefore, evaluating a process given by an indicator set $I$ and relation $R$ becomes different, in fact finer", if one evaluated via the aspect relation (of course one of the main reasons to undertake aspect evaluation in the first place). 
Terminology for $d w(A, B)$ corresponds to the fact that $A R B$ holds for $A \neq B$ when $\delta_{q}(A \times B)>0$. We also introduce the gross dominant weight by putting this equal to $w(A) w(B) d w(A, B)$ and denoting this by $W(A, B)$. Observe that we may apply all constructions to $(I, R)$ with respect to any given function $W: I \rightarrow \mathbb{R}_{+}$, leading to a new relative weight expressed by : $W(A) W(B) t(A)^{-1}$ with $t(A)=\sum_{T(A)} W(A) W(X)$. If $W$ derives from $w$ and $q$ induces $R$, even then the foregoing is different from the dominant weight $W(A, B)$, because again the latter is calculated from aspect relations as explained above. Another type of variation on the theme of indicator weights stems from a practical observation that in nature, perhaps it is better to say in concrete examples, the process or some of its ingredients can be in a certain "state", e.g., there may be a switch function on-off as in many industrial or engineering processes, or there may be a system of fine-tuning and a gradation in the intensity of process activity. To some extent this is similar to the introduction of probabilities (see 2.1) but "state" is a more discrete notion and the interaction between ingredients of the process in different states behaves differently (not based on multiplicativity).

\subsection{Actions on processes}

Let us give a state function $\Psi$ on the aspect set, $\Psi: \mathcal{A} \rightarrow \mathbb{Z}$. We allow the integers $\mathbb{Z}$ even if it is clear that in many concrete applications we may work with a positive function $(\Psi: \mathcal{A} \rightarrow \mathbb{N})$ but we need not restrict to that here. Because of the interpretation we aim to give at such a state function, we define the graph-state function $\Psi_{g}: G(q) \rightarrow \mathbb{Z}$ as the restriction of $\mathcal{A} \times \mathcal{A} \rightarrow \mathbb{Z}$ mapping $(a, b)$ to $\min \{\Psi(a), \Psi(b)\}$.

Any state function allows to modify the value function $w$ to $\Psi w$ and the relative weight associated to $w$, that is, $w(a, b)$, to $\Psi_{q}(a, b) w(a, b)$. Moreover, we may induce an indicator state function $\Psi_{i}: I \rightarrow \mathbb{Z}$ from $\Psi$ by putting : $\Psi_{i}(A)=\min \{\Psi(a), a \in A\}$. All earlier constructions may now be applied to the active value $\Psi w$ instead of $w$ and this will yield active versions of all objects and function defined before, in particular, we 
arrive at the active relation $R^{\Psi}$ on $I$. Observe though, that in case nonpositive state functions are used in $\mathbb{R}$, not in $\mathbb{R}_{+}$, but that is harmless for most properties.

Up to minor modifications related to a possible non-positivity of the state function, both the state function and active weight function allow diagonalization in the way described earlier. However, we provide here a different way, taking into account our interpretation of $\Psi$ as a process state. For indicators $A, B$ in $I$, we write: $\Psi(A, B)=\min \left\{\Psi_{q}(a, b), a \in A\right.$, $\left.b \in B\}-\min \Psi_{q}(b, a), a \in A, b \in B\right\}$. In case $A R B$ holds exactly when $\Psi(A, B)>0$, then we say that $R$ is in active state. Observe however that, even if $R$ is in active state, we do not necessarily have that $R^{\Psi}$ is equal to $R$; indeed, the "activity level" of certain aspects (given by the value of the state function) having high $w$-values may easily change the dominant weight and then also the relation induced on $I$. Many interesting questions of algebraic or combinatorial nature arise from the problem of controlling the variation of $R^{\Psi}$ and its comparison to $R$, it is well possible to develop examples using computer simulation or even based on more old-fashioned lamp-systems used by engineers as didactical models of elementary production processes. We do not develop a deeper theory about $R^{\Psi}$ here. Instead we define that $R$ is in core state if it is in active state such that $R=R^{\Psi}$. The $q$-core of the process described by $(\mathcal{A}, q)$ is the set of all functions $\Psi: \mathcal{A} \rightarrow \mathbb{N}$ leaving $R$ in core state. Any given state function $\varphi: I \rightarrow \mathbb{Z}$ defines in a canonical way a state function $\varphi^{a}: \mathcal{A} \rightarrow \mathbb{Z}$ by putting $\varphi^{a}(x)=\varphi(X)$, where $X \in I$ is such that $x \in X$. Starting with $\varphi=\Psi_{i}$ we arrive at $\varphi^{a}=\Psi^{a}$. If $\varphi$ is positive and $\Psi$ is in the $q$-core, then $\varphi^{a} \Psi$ is in the $q$-core too. In general, any state function $\phi: \mathcal{A} \rightarrow \mathbb{Z}$ such that $\Psi \phi$ is in the $q$-core is said to be in the $\Psi$-stabilizer; in case $\phi$ stabilizers every $\Psi$ in the $q$-core the $\phi$ is said to be $q$-stabilizing. A trivial example of a $q$-stabilizing function is obtained by taking any constant positive function. 


\subsubsection{Process tuning}

In the foregoing $\mathbb{Z}$ may be replaced by any totally ordered set or group, in particular any totally ordered group embedded in $\mathbb{R}_{+}^{n}$ (additive group of $\mathbb{R}^{n}$ ), say $\Gamma$. This allows a more continuous way of using states and a more natural notion of "tuning", i.e., manipulation of state functions $\Psi$ such that $R$ is in core state and studying the effect of $q$ stabilizing changes of the state function on other properties of the process. If suitable $\Gamma$ are used instead of $\mathbb{Z}$ a micro- or infinitesimaltuning can be defined. We leave it as a project for now to work out (using computer simulation) some explicit cases. This aspect of the theory is very related to process interaction (e.g., inspection processes) dealt with later in this text.

\subsubsection{Aspect changes}

Viewing different sets of aspects as subsets of the union, most "changes of aspects" correspond to restricting some set of aspects to suitable subsets. Even a dynamic theory fits in this scope (see hereafter)! Give for each $t \in \mathbb{R}$ an aspect set $\mathcal{A}_{t}$ with indicator set $I_{t}$. In the context of process evaluation certain intuitive rules govern the variation of aspects, e.g., an aspect of a given indicator should not turn into an aspect of another indicator, at least not in a "small" time interval, etc. We formalize a desirable set of rules in the following definition of a process dynamics.

For every $t \leq t^{\prime}$ in $\mathbb{R}$, we suppose given maps defined on a subset $\dot{\mathcal{A}}_{t}$ of $\mathcal{A}_{t}, \varphi_{t, t^{\prime}}: \dot{\mathcal{A}}_{t} \rightarrow \mathcal{A}_{t^{\prime}}$, satisfying the following conditions:

D.1. If $B_{t} \in I_{t}$, then for $b_{t} \in B_{t}$ either $\varphi_{t, t^{\prime}}\left(b_{t}\right)$ is not defined or $b_{t} \in \dot{\mathcal{A}}_{t}$ and $\varphi_{t, t^{\prime}}\left(b_{t}\right) \in B_{t^{\prime}}$. Hence, if $\pi_{t}^{-1}\left(B_{t}\right) \cap \mathcal{A}_{t} \neq \varnothing$, then $B_{t^{\prime}} \in I_{t^{\prime}}$ is uniquely determined as $\pi_{t^{\prime}}\left(b_{t^{\prime}}\right)$, where $b_{t^{\prime}}=\varphi_{t, t^{\prime}}\left(b_{t}\right)$.

D.2. For $t \leq t^{\prime} \leq t^{\prime \prime} \in \mathbb{R}$, we have $\varphi_{t, t^{\prime \prime}}=\varphi_{t^{\prime}, t^{\prime \prime}} \circ \varphi_{t, t^{\prime}}$, whenever the first one has a defined value. 
D.3. For $t_{1} \leq t$ either $\varphi_{t_{1}, t}^{-1}\left(\pi_{t}^{-1}\left(B_{t}\right)\right)$ is empty or contained in some $\pi_{t_{1}}^{-1}\left(B_{t_{1}}\right)$ for $B_{t_{1}} \in I_{t_{1}}$, then $B_{t_{1}}$ is uniquely determined.

D.4. In case $t^{\prime} \geq t$ and $x \in \mathcal{A}_{t}, x \in \mathcal{A}_{t^{\prime}}$, then we have an identity principle stating that $\varphi_{t, t^{\prime}}(x)=x$, meaning in particular that $\varphi_{t, t^{\prime}}$ is defined on $x$, in other words $x \in \dot{\mathcal{A}}_{t^{\prime \prime}}$ for all $t \leq t^{\prime \prime} \leq t^{\prime}$ (see also D.2).

For notational convenience, let us agree to write $\varphi_{t, t^{-1}}\left(B_{t}\right)$ for $\varphi_{t, t^{\prime}}\left(\pi_{t}^{-1}\left(B_{t}\right)\right)$ consistent with viewing an indicator as the set of its aspects. Also, we have written $\dot{\mathcal{A}_{t}}$ as domain of definition for $\varphi_{t, t^{\prime}}$ and in principle there is another subset of $\mathcal{A}_{t}$ to be considered for each $t^{\prime}, t \leq t^{\prime}$, this does not create ambiguity except perhaps in D.2, where one has to read the equality up to the condition that all maps are defined on the arguments. In fact, one may work with everywhere defined $\varphi_{t, t^{\prime}}, t \leq t^{\prime}$ in $\mathbb{R}$, at the cost of adding formally the empty set to the partition of $\mathcal{A}_{t}$ indexed by $I_{t}$, this may be understood as adding a superuous aspect to each $\mathcal{A}_{t}$ and to extend the original $\varphi_{t, t^{\prime}}$ to $\mathcal{A}_{t} \cup\{\varnothing\}$ by sending $\{\varnothing\}$ to $\{\varnothing\}$ and also the $a_{t}$, where $\varphi_{t, t^{\prime}}$ is not defined are mapped to $\{\varnothing\}$ by the extension $\bar{\varphi}_{t, t^{\prime}}$. This is obviously a non-essential modification but it allows us to consider dynamical processes where some aspects disappear in time (the $\varphi_{t, t^{\prime}}$ not being surjective "new" aspects may exist at moment $t^{\prime}$ in the sense that they do not come from an aspect at moment $t$ ).

Put $\underline{\mathcal{A}}=\bigcup\left\{\mathcal{A}_{t}, t \in \mathbb{R}\right\}$. For $B_{t} \in I_{t}$, we look at $\underline{B}_{t}=\bigcup\left\{B_{t^{\prime}} \in I_{t^{\prime}}\right.$ such that either $\varphi_{t, t^{\prime}}\left(B_{t^{\prime}}\right) \subset B_{t}$ or $\varphi_{t, t^{\prime}}\left(B_{t^{\prime}}\right) \subset B_{t^{\prime}}$ depending whether $t^{\prime} \leq t$ or $t \leq t^{\prime}$, resp. Note that the assumptions, cf. D.4, imply that $\varphi_{t, t}$ is the identity of $\mathcal{A}_{t}$, hence we always have $B_{t} \subset \underline{B}_{t}$ for every $t \in \mathbb{R}$. Observe 
that $\underline{B}_{t}=B_{t}$ is possible (when $B_{t} \cap \dot{\mathcal{A}_{t}}=\phi$ ). But also note that neither $\underline{\mathcal{A}}$ nor $\underline{B}_{t}$ is given as a collection of elementwise strings $\left(\ldots a_{t}, \ldots, a_{t^{\prime}} \ldots\right)$ such that $\varphi_{t, t^{\prime}}\left(a_{t}\right)=a_{t^{\prime}}$ for all $t \leq t^{\prime}$ in $\mathbb{R}$; this is possible but leads to other processes.

Proposition 2.5.3. With notation as above and assuming D.1. . D.4:

(1) If $B_{t^{\prime}} \subset \underline{B}_{t}$, then $\underline{B}_{t^{\prime}}=\underline{B}_{t}$.

(2) For $t \in \mathbb{R}, \underline{B}_{t} \cap \mathcal{A}_{t}=B_{t}$.

(3) The set of all $\underline{B}_{t}$ defines a partition of $\underline{\mathcal{A}}$ such that for each $t \in \mathbb{R}$ it induces $I_{t}$ in $\mathcal{A}_{t}$.

Proof. If $x \in \underline{\mathcal{A}}$, then $x \in \mathcal{A}_{t}$ for some $t \in \mathbb{R}$ and hence $x \in B_{t}$ for some $B_{t} \in I_{t}$, or $x \in \underline{B}_{t}$ defined by $B_{t}$.

(1) Look at $A_{t^{\prime}} \in I_{t^{\prime}}$ and suppose that $A_{t^{\prime}} \subset \underline{B}_{t}$. There are two cases to consider : $t^{\prime} \leq t$ or $t \leq t^{\prime}$. First consider the case $t \leq t^{\prime}$ then $A_{t^{\prime}} \subset \underline{B}_{t}$ means that $\varphi_{t, t^{\prime}}\left(B_{t}\right) \subset A_{t^{\prime}}$ and $\varphi_{t, t^{\prime}}\left(B_{t}\right) \neq \phi$. It follows that

$$
\phi \neq B_{t} \cap \varphi_{t, t^{\prime}}^{-1}\left(A_{t^{\prime}}\right) \text { thus } \varphi_{t, t^{\prime}}^{-1}\left(A_{t^{\prime}}\right) \neq \phi .
$$

In view of D.3, there is a unique $A_{t}$ in $I_{t}$ such that $\varphi_{t, t^{\prime}}^{-1}\left(A_{t^{\prime}}\right) \subset A_{t}$, but since $B_{t}$ contains an element from $\varphi_{t, t^{\prime}}^{-1}\left(A_{t^{\prime}}\right)$ it follows that $A_{t}=B_{t}$. Secondly, look at the case $t^{\prime}<t$, then $A_{t^{\prime}} \subset \underline{B}_{t}$ means $\phi \neq \varphi_{t, t^{\prime}}\left(A_{t^{\prime}}\right) \subset B_{t}$, so if $A_{t}$ denotes the unique indicator in $I_{t}$ containing $\varphi_{t, t^{\prime}}\left(A_{t^{\prime}}\right)$ (see D.1), then $A_{t}=B_{t}$. In both cases, we see that $B_{t} \subset \underline{A}_{t^{\prime}}$ and thus $\underline{B}_{t} \subset \underline{A}_{t^{\prime}} \subset \underline{B}_{t}$ trivially follows from $A_{t^{\prime}} \subset \underline{B}_{t}$.

(2) Look at $b \in \underline{B}_{t} \cap \mathcal{A}_{t}$. Then $b \in B_{t_{1}} \subset \mathcal{A}_{t_{1}}$ for some $t_{1} \in \mathbb{R}$. In case $t_{1} \geq t$, then we have : $\varphi_{t, t_{1}}(b)=b \in B_{t_{1}}$ (see D.4) with $b \in A_{t}$. 
Therefore $t=t_{1}$ and $b \in B_{t}$. On the other hand, when $t_{1} \leq t$, then $\varphi_{t_{1}, t}(b)=b$ hence $b \in \varphi_{t_{1}, t}\left(B_{t_{1}}\right) \subset B_{t}$ (see D.1) or $b \in B_{t}$. The fact that $B_{t} \subset \underline{B}_{t}$ yields $B_{t}=\underline{B}_{t} \cap \mathcal{A}_{t}$.

(3) Look at different $\underline{A}_{t^{\prime}}$ and $\underline{B}_{t}$; in view of (1), we may write these as $\underline{A}_{t}$ and $\underline{B}_{t}$ with $A_{t}$ and $B_{t}$ in $I_{t}$. If $x \in \underline{A}_{t} \cap \underline{B}_{t}$, then for some $t_{1} \in \mathbb{R}, x \in B_{t_{1}}$ in $I_{t_{1}}$, and for some $t_{2} \in \mathbb{R}, x \in A_{t_{2}}$ in $I_{t_{2}}$. Suppose $t_{2} \leq t_{1}$ (otherwise reverse the roles of $t_{1}$ and $t_{2}$ and $A$ and $B$ ). Then $\varphi_{t_{2}, t_{1}}(x)=x$ (see D.4) yields $x \in \varphi_{t_{2}, t_{1}}\left(A_{t_{2}}\right)$ with $x$ also being in $B_{t_{1}}$, thus $A_{t_{1}}$ being the unique indicator such that $\varphi_{t_{2}, t_{1}}\left(A_{t_{2}}\right) \subset A_{t_{1}}$ (see D.1), it follows that $B_{t_{1}}=A_{t_{1}}$ hence $\underline{B}_{t_{1}}=\underline{A}_{t_{1}}$. Applying (1) to $B_{t_{1}} \subset \underline{B}_{t}$ and $A_{t_{1}} \subset \underline{A}_{t}$ yields $\underline{B}_{t}=\underline{A}_{t}$.

In concrete situations, most processes have a starting point $t_{0}$ in $\mathbb{R}$, or else at least a specific moment of observation is defined. Then it makes sense to consider $\underline{\mathcal{A}}_{0}=\bigcup\left\{\underline{B}_{t_{0}}\right.$, all $B_{t_{0}}$ in $\left.I_{t_{0}}\right\}$. This is the set of all aspects related *by strings) in time to aspects in $\mathcal{A}_{t_{0}}$. The process described by $\underline{\mathcal{A}}_{0}$ will contain the essential information about local properties at the moment $t_{0}$, so if one is primarily interested in those local properties it is natural to restrict from $\underline{\mathcal{A}}$ to $\underline{\mathcal{A}}_{0}$. This is one example of "restricting of aspects" which we deal with further on.

So far we neglected the relations defining the partitions, in order to deal with variations in the relations we now will add an axiom D.5 dealing with this problem.

\section{Definition 2.5.4. (dynamical process)}

D.5. If $q_{t}$ is the relation considered on $\mathcal{A}_{t}$, then we demand $\varphi_{t, t^{\prime}} \times \varphi_{t, t^{\prime}}\left(q_{t}\right) \subset q_{t^{\prime}}$, i.e., if $a_{t} q_{t} b_{t}$ in $\mathcal{A}_{t}$, then $\varphi_{t, t^{\prime}}\left(a_{t}\right) q_{t^{\prime}} \varphi_{t, t^{\prime}}\left(b_{t}\right)$ in $\mathcal{A}_{t^{\prime}}$. Then we call $\left(\mathcal{A}_{t}, B_{t}\right)$ a dynamical process. 
By definition of a dynamical process, the $\varphi_{t, t^{\prime}}$ define $\bar{\varphi}_{t, t^{\prime}}: I_{t} \rightarrow I_{t^{\prime}}$ such that the following diagram is commutative:

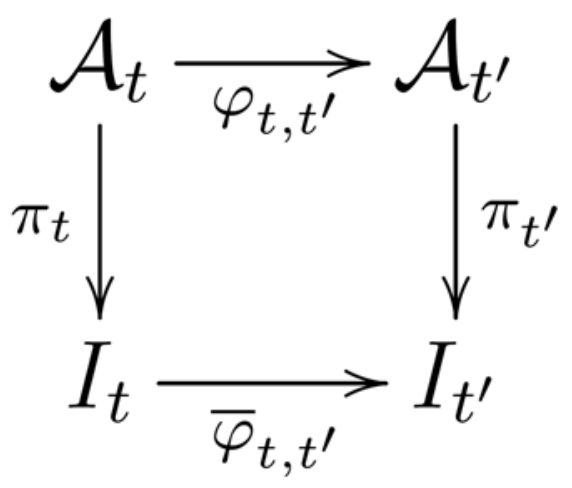

Note however that we need not have that $\left(\bar{\varphi}_{t, t^{\prime}} \times \bar{\varphi}_{t, t^{\prime}}\right)\left(R_{t}\right) \subset R_{t^{\prime}}$, where $R_{t}$ and $R_{t^{\prime}}$ are derived from $q_{t}$ resp., $q_{t^{\prime}}$ via certain value function.

Interesting value functions are globally defined, e.g., $\underline{w}: \underline{\mathcal{A}} \rightarrow \mathbb{R}$, and restrict to $w_{t}: \mathcal{A}_{t} \rightarrow \mathbb{R}$. Even then, with respect to $\left(\mathcal{A}_{t}, B_{t}, w_{t}\right)$ and the defined relation $R_{t}$ on $I_{t}$, we do not necessarily obtain a dynamical system $\left(I_{t}, R_{t}\right)$ given by the $\bar{\varphi}_{t, t^{\prime}}$. Indeed deleting "important" aspects, i.e., with large value for $w$ may destroy the diagonal weight such that $\bar{\varphi}_{t, t^{\prime}}$ do not respect the induced relations. By controlling $\left|w_{t^{\prime}} \varphi_{t, t^{\prime}}\left(a_{t}\right)-w_{t}\left(a_{t}\right)\right|$ uniformly for $t^{\prime}$, one may arrive at value functions for which $\left(\bar{\varphi}_{t, t^{\prime}} \times \bar{\varphi}_{t, t^{\prime}}\right)\left(R_{t}\right) \subset R_{t^{\prime}} \forall t \leq t^{\prime}$ in $\mathbb{R}$.

Then we say that $(\mathcal{A}, B, w)$ is an indicator induced dynamical process. In our approach without convergence conditions on weight, we shall assume $\underline{\mathcal{A}}$ is finitary (e.g., one may just be interested in processes described by finitely many aspects, as in practice!).

In view of Proposition 2.5.3, any dynamical process may be viewed as a process defined on $\underline{\mathcal{A}}=U_{t}\left\{\mathcal{A}_{t}, t \in \mathbb{R}\right\}$. Since we may allow infinitely many $t \in \mathbb{R}$, even if all processes defined at $t \in \mathbb{R},\left(\mathcal{A}_{t}, I_{t}\right)$ are finite, 
then $\underline{\mathcal{A}}$ is not finite (but it is again finitary). In any case, a dynamical process satisfying the conditions D.1, .., D.5 may be viewed as a unique global process on $\underline{\mathcal{A}}$.

\subsubsection{Aspect restriction}

An indicator induced dynamical process may be studied by looking at restrictions of $\underline{\mathcal{A}}$ to the subsets $\mathcal{A}_{t}$, where a particular $\underline{I}$ of $\underline{\mathcal{A}}$ induces the partition $I_{t}$ of $A_{t}$ and $R_{t}$ on $\mathcal{A}_{t}$ is induced by a relation $\underline{R}$ on $\underline{\mathcal{A}}$. Therefore, let us now look at $\mathcal{B} \subset \mathcal{A}$. For $A \in I$, we define $A_{\mathcal{B}}=A \cap \mathcal{B}$, $\left|A_{\mathcal{B}}\right|=m_{A} \leq n_{A}$. If $m_{A} \neq 0$, then we say $A \in I_{\mathcal{B}} \subset I$. Obviously, we have : $\bigcup\left\{A_{\mathcal{B}}, A \in I_{\mathcal{B}}\right\}=\bigcup\{A \cup \mathcal{B}, A \in I\}=\mathcal{B}$ and nonempty $A_{\mathcal{B}}$ form a partition of $\mathcal{B}$. The restriction of $w: \mathcal{A} \rightarrow \mathbb{R}$ defines $w \mid \beta: \mathcal{B} \rightarrow \mathbb{R}$, which we denote again by $w$. For $A \in I_{\mathcal{B}}$, we have now $w(A)$ but also $w_{\mathcal{B}}(A)=\sum_{A \cap \mathcal{B}} w(x)$. The relation induced in $\mathcal{B}$ by a relation $R$ on $\mathcal{A}$ is again denoted by $R$. Similar to $w(a, b)$, we now also define $w_{\beta}(a, b)$ $=w(a) w(b)\left(t_{\mathcal{B}}(a)\right)^{-1}$, where now $t_{\mathcal{B}}(a)=\sum_{\mathcal{B} \cap T(a)} w(a) w(x)$, for $a$ and $b$ in $\mathcal{B}$. Analogously, one may introduce the historic $\mathcal{B}$-weight $h_{\beta}(a, b)$ for $a, b$ in $\mathcal{B}$, as well as a $\mathcal{B}$-version of all weights and functions defined earlier. Let us just point out what happens to the graph-and diagonal weight. Consider $S \subset \mathcal{B} \times \mathcal{B} \subset \mathcal{A} \times \mathcal{A}$, look at $S \cap G(q)$ and define

$$
w_{R, \mathcal{B}}(S)=\left(\sum_{S \cap G(q)} w\left(b_{1}\right) w\left(b_{2}\right)\right)\left(\sum_{S} w\left(b_{1}\right) w\left(b_{2}\right)\right)^{-1}
$$

(formally the same as $w_{q}(S)$ ) because we restricted attention to $S \subset \mathcal{B} \times \mathcal{B}$. For $S=A \times B \subset \mathcal{A} \times \mathcal{A}$ with $A, B$ in $I$, we now obtain

$$
w_{q, \mathcal{B}}(A, B)=\left(\sum_{G(q) \cap((\mathcal{B} \cap A) \times(\mathcal{B} \cap B))} w\left(b_{1}\right) w\left(b_{2}\right)\right) w_{\mathcal{B}}^{-1}(A) w_{\mathcal{B}}^{-1}(B) .
$$

We now use $w_{R, \mathcal{B}}$ to define $\delta_{q, \mathcal{B}}$ and a new relation $R_{\mathcal{B}}$. 
In general, $R$ and $R_{\mathcal{B}}$ are not too nicely related but under suitable conditions the situation becomes better. We say that $\mathcal{B}$ is a defining subset of $\mathcal{A}$ whenever $A R B$ if and only if $A R_{\mathcal{B}} B$, i.e., when $R=R_{\mathcal{B}}$. Passing from $\mathcal{A}$ to a defining subset may be thought of as dumping unnecessary aspects in the sense that they are superuous in detecting the intrinsic relation. On the other hand, $\mathcal{A}$ is said to be I-perfect if there is no $\mathcal{B} \subseteq \mathcal{A}$ such that $\mathcal{B}$ is a defining subset of $\mathcal{A}$. In case we also pass to a subset of indicators, i.e., $I^{\prime} \subseteq I$ then $\mathcal{A}^{\prime}=U\left\{A, A \in I^{\prime}\right\}$ is defining subset for $I^{\prime}$.

A minimal (with respect to inclusion) defining subset of $\mathcal{A}^{\prime}$ (for $I^{\prime}$ ) is necessarily $I^{\prime}$-perfect, and the existence of such minimal subsets is guaranteed in the finitary case. There one many interesting problems related to the dynamical theory, the search for $I$-perfect $\mathcal{A}$ in describing a process has an economic meaning as it is cost reducing (where cost can be time or money!).

\subsubsection{A practical procedure diagram}

This short section presents a practical schematic way to present the aspect-deformation of an indicator ordering process. We consider the indicator set $I$ and compose an aspect set $\mathcal{A}$ containing $k$ different classes : $K_{1}, \ldots, K_{k}$. For $A \in I$, we denote $A=\left\{a_{i_{1}}, \ldots, a_{i_{k}}\right\}$, where $a_{i_{j}} \in K_{j}$, for $i=1$ we have a first layer of elements $a_{1_{s}}, \ldots, a_{1_{k}}$, where if some class ( $j$ say) does not have an aspect in it $a_{1}$ is supposed to be "empty", again for $i=2, \ldots$ until all aspects have been layered and some $a_{i_{j}}$ being empty. The total: $|A|=n_{A}$. Positive arrows are drawn as full arrows between aspects, "negative"-arrows as a dotted arrow. In the column $I$, we put on the first line the primary indicators, on the second line the indicators only having primary indicators as smaller once (i.e., with respect to $\leq), \ldots$, on the $n$-th-line the indicators having as predecessors only the indicators appearing in the $n-1$ foregoing lines, etc. The column $\mathcal{A}$ is subdivided in $K_{1}, \ldots, K_{k}$. 
On the first line in the $\mathcal{A}$ column, we put horizontally the aspects of the indicators in the corresponding indicators from the $I$-column (reading from left to right) ordered according to the classes and using the notation introduced above; underneath every aspect we may write its $w$-value.

In this organization, positive arrows run from top to bottom and negative arrows run upwards. Observe that the arrangement chosen for $I$ defines the height of an indicator $h|A|$ for $A \in I$; that is $h(X)=1$ for primary indicators and $h(X)=1$ means that for $Y \leq X$ with $Y \neq X$ we necessarily have $Y$ primary. Hence, we put $X$ at the height of the length of an extremal path from a primary indicator to $X$. The height of the scheme is therefore at most equal to the length of an extremal path of $I$ (a path of maximal length in $I$ ). This arrangement also has the advantage that in the $I$-column, no horizontal arrows exists, hence in the $\mathcal{A}$-column no horizontal positive (full line) arrows exist. The scheme we obtain provides a standardized way of dealing with not too big process on paper, for large processes it may be treated on computer. For example:

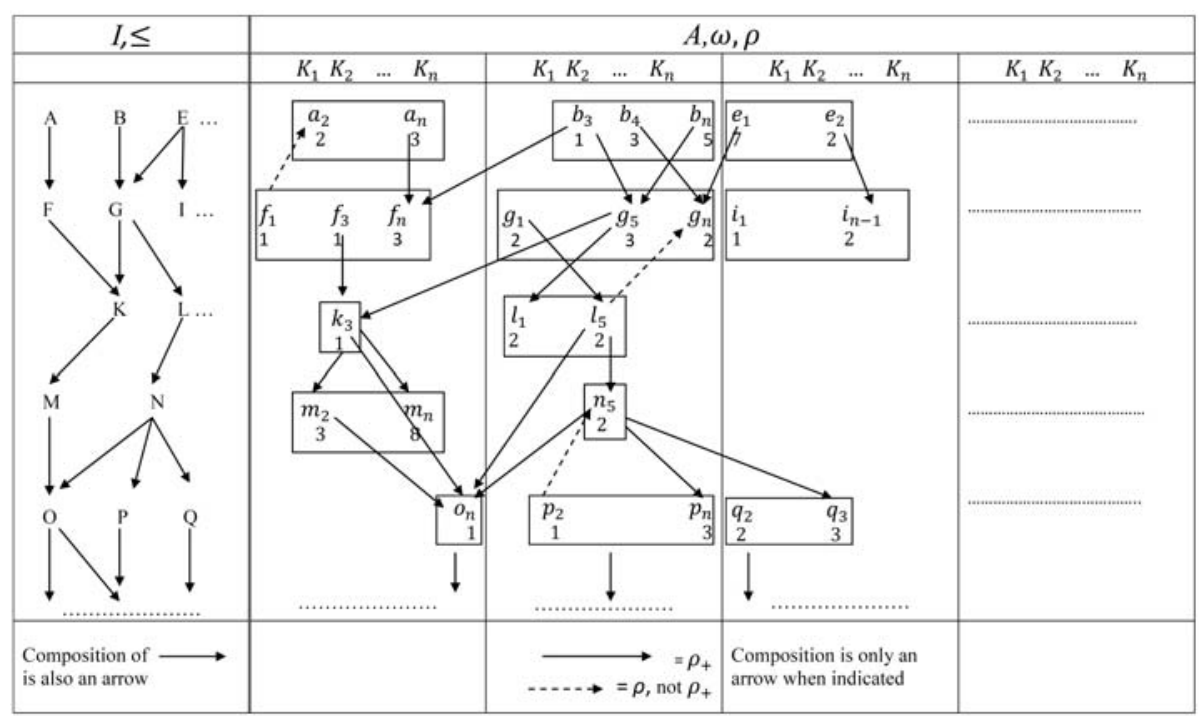




\section{Some Remarks on Applications and Work in Progress}

\subsection{Some existing applications}

The idea of this paper was to introduce a new generic and measurable description of processes via the aspect deformation of a relation. We came to this idea via a practical problem, i.e., the evaluation of the IMPACTproject of the Flemish Ministry of Education (2002-2003) [4]. Via a wellconstructed questionnaire, the process of "effects of internationalization on the curricula of Flemish institutions of higher education" could be evaluated. The evaluation of the process followed from a well-chosen weighing of the branches in the indicator process. After this, the generic property of the approach via the aspect deformation of the indicator process became clear and this was first applied in some local experiments in didactics with students of the University of Antwerp during their teacher-practice in secondary schools. The learning process as a deformation of a study program is the topic of a forthcoming paper [5]. Another application of the new structure is in the description of the (very complex) creative process as a deformation of a causal cognitive process where the aspect relation contains non-causal relations (deriving from the use of fantasy and emotional aspects) appearing in what we called the "transfer" activity [1].

A very abstract application is the definition of a noncommutative topology (geometry) as an aspect deformation of a commutative topology (geometry) [6]; this leads to new mathematical theories. This is an abstract application but it shows the generic quality of the new structure.

Now there are possible applications in almost all sciences, we mention a few ideas, some of these outside the area of our expertise, just to show the many doors that open for possible applications and new research. 


\subsection{Some suggestions}

\subsubsection{Microstructure of biodiversity}

The indicator process is described by the evaluation of the biological activity of species in some biotope including their genetic information and their behavioural mode. The aspects are obtained from measurements in the biotope, properties of soil or environment, presence of minerals, plant life, pollution level, climate factors, etc. The weighing is given by a scientific measurement of the effect of the aspect on the ingredient and it induces an indicator relation via the relation on aspects. One may study allowable variation in the measurements in order to leave the indicator relation unchanged. When the indicator relation changes over different measurements, positive or negative effects changes in weighing of the aspect relation become clear and this provides insight in the microstructure of the biodiversity, e.g., the effect of acid rain on the weighing of the aspect relation.

\subsubsection{Safety and security issues of a production process}

The indicator process describes the production of an item, the aspects describe the local conditions locally at each production step, e.g., pressure, temperature, humidity, radiation level. The weighing is a measurement of the process in a safe situation. The aspect relation provides insight in the micro-process of the production with influence from internal and external factors. Manipulation of the weighing allows to see the possible changes in the production process and predict its effects on safety.

\section{References}

[1] M. Ma, The Creative Process as an Aspect Deformation and Creative Transformation in Learning Process, Ph.D. Thesis, University of Antwerp, Antwerp, 2013.

[2] F. Van Oystaeyen, Noncommutative space from dynamical noncommutative geometries, Journal of Geometry and Physics 2(59) (2009), 185-196.

DOI: http://dx.doi.org/10.1016/j.geomphys.2008.10.002. 
[3] M. Gerstenhaber and S. Schack, Algebraic Cohomology and Deformation Theory, Springer Netherlands, Deformation Theory of Algebras and Structures and Applications 247 (1988), 11-264.

[4] F. De Decker, An evaluation of the impact of internationalization on the higher education curricula (in anders), Tech. Rep., Report of the Project "IMPACT" of the Flemish Ministry of Education, 2002.

URL http://www.arteveldehs.be/onet/projecten/impact.htm

[5] M. Ma and F. Van Oystaeyen, A learning process as a deformation of a study program, [submitted].

[6] F. Van Oystaeyen, Virtual Topology and Functor Geometry, Lecture Notes in Pure and Applied Mathematics, Chapman and Hall/CRC, 2007. 